\title{
Ultrasound visibility of regional anesthesia catheters: an in vitro study
}

\author{
Junji Takatani, Naozumi Takeshima, Kentaro Okuda, Tetsuya Uchino, and Takayuki Noguchi \\ Department of Anesthesiology and Critical Care Medicine, Oita University Faculty of Medicine, Oita, Japan
}

Background: Ultrasound subjective visibility of in-plane needles is correlated with the intensity difference between the needle surface and the background. Regional anesthesia catheters are difficult to visualize by an ultrasound. In the present study, we investigated the ultrasound visibility of the catheters.

Methods: Six catheters were placed at $0^{\circ}$ and $30^{\circ}$ relative to and at a depth of $1 \mathrm{~cm}$ below the pork phantom surface. Ultrasound images of in-plane catheters were evaluated, subjectively and objectively. Outer and inner objective visibilities were defined as the difference in the mean pixel intensity between the catheter surface and adjacent background, and between the surface and the center of the catheter, respectively. Evaluations were made based on the portion of the catheters. A $P$ value $<0.05$ was considered significant.

Results: Subjective visibility was more strongly correlated with the inner objective visibility than with the outer objective visibility at both angles. Metallic 19-gauge catheters were more subjectively visible than the non-metallic 20 -gauge catheters at $30^{\circ}$ degrees $(\mathrm{P}<0.01)$. Subjective, and outer and inner objective visibility were significantly lower at $30^{\circ}$ than at $0^{\circ}(\mathrm{P}<0.01, \mathrm{P}<0.01, \mathrm{P}=0.02)$. Perifix ONE at $0^{\circ}$ and Perifix FX at $30^{\circ}$ were the most visible catheters ( $\mathrm{P}<0.01$ for both).

Conclusions: Subjective visibility of catheters can not be evaluated in the same manner as that of the needles. For the best possible visualization, we recommend selecting a catheter with a structure that enhances the dark at the center of catheter, rather than basing the catheter selection on the bore size. (Korean J Anesthesiol 2012; 63: 59-64)

Key Words: Catheters, Nerve block, Regional anesthesia, Ultrasonography.

Received: March 23, 2012. Revised: March 26, 2012. Accepted: June 8, 2012.

Corresponding author: Junji Takatani, M.D., Department of Anesthesiology and Critical Care Medicine, Oita University Faculty of Medicine, 1-1 Idaigaoka-Hasamamachi, Yufu City, Oita 879-5593, Japan. Tel: 81-97-586-5943, Fax: 81-97-586-5949, E-mail: jt205050@oita-u.ac.jp (c) This is an open-access article distributed under the terms of the Creative Commons Attribution Non-Commercial License (http:// creativecommons.org/licenses/by-nc/3.0/), which permits unrestricted non-commercial use, distribution, and reproduction in any medium, provided the original work is properly cited. 


\section{Introduction}

There have been several studies on the ultrasound visibility of non-textured needles for the use in regional anesthesia $[1,2]$. Ultrasound-guided nerve blocks performed with non-textured needles have better success rates than that of the traditional methods of nerve blocking, such as fluoroscope-guided, and landmark approach [3-7]. The subjective visibility of in-plane needles is reported to be correlated with the intensity difference between the needle surface and the background [8].

Although conventional regional anesthesia catheters need to be positioned precisely beside the target nerve, they are difficult to visualize by ultrasound [9-11]. The relative ultrasound visibility of commonly used catheters has not been previously reported. In the present study, we assessed the ultrasound visibility of a variety of catheters, and determined the impact of catheter width and angle on the visibility in vitro.

\section{Materials and Methods}

\section{Catheters}

We examined 6 catheters that are commonly used for ultrasound-guided regional anesthesia in Japan (Table 1).

\section{Ultrasound equipment}

We used a nerve block-specific ultrasound device S-Nerve, with linear probe: HFL38x 13-6 MHz, (SonoSite Inc., Bothell, WA, USA) in this study, with the examination type set to "Nerve" mode. The gain was not adjusted after start-up, and neither were any other configurations.

\section{Phantom}

Because anatomic structures of a pork phantom are clearly visualized with an ultrasound, including the background echogenicity, tissue layers, and needle images that are com-

Table 1. Characteristics of Catheters

\begin{tabular}{lcll}
\hline Catheter & $\begin{array}{c}\text { Diameter } \\
\text { (gauge) }\end{array}$ & \multicolumn{1}{c}{$\begin{array}{c}\text { Material } \\
\text { (outer layer/core) }\end{array}$} & Manufacturer \\
\hline Perifix ONE & 20 & Polyurethane/polyamide & B Braun \\
Soft tip & 20 & Polyamide & B Braun \\
Nipro & 20 & Nylon & Nipro \\
Peribax & 20 & Polyurethane & Vygon* \\
Flex tip plus & 19 & Polyurethane/stainless coil & Arrow \\
Perifix FX & 19 & Polyamide/stainless coil & B Braun \\
\hline
\end{tabular}

Catheters commonly used for ultrasound-guided regional anesthesia in Japan. *Supplied to Unisis (Tokyo, Japan) as an original equipment manufacturing and saled as Uniset in Japan. parable to those in the human tissue [12], we used a boneless pork phantom $(10 \times 10 \times 20 \mathrm{~cm})$.

\section{Study protocol}

Catheters were primed with saline. We placed catheters at $0^{\circ}$ and $30^{\circ}$ relative to the pork phantom surface at a depth of 1 $\mathrm{cm}$ below the upper surface of the pork phantom to the probe surface. All catheters were inserted in-plane with a needle guide (Infiniti, CIVCO Medical Solutions, Kalona, IA, USA), using the needles provided in the respective catheterization kits. We avoided prior traces when inserting the catheters. Each catheter was placed as straight as possible to minimize the influence of winding on the visibility. All images of the catheters were digitally recorded to the hard disk to analyze at a later time. We inserted 10 catheters of each type into the pork phantoms, using this procedure.

\section{Image evaluation}

Assessment of the acquired images was performed using Photoshop Elements (Version 7.0; Adobe Systems, San Jose, CA, USA) by an investigator who was not involved to the blinding of the catheter type. Images were placed in random order, and the ultrasound visibility was evaluated according to the methods described by Schafhalter-Zoppoth et al. [8], with modifications. Pixel intensity was defined as the gray-scale value between 0 (black) and 255 (white). The outer objective visibility (pixel intensity units [PIUs]) was estimated by the difference in the mean pixel intensity between the surface of the catheter and the adjacent background. We also measured the outer objective visibility of the brightest tissue on each image. We defined the inner objective visibility as the difference in the mean pixel intensity between the surface and the center of the catheter. Negative values indicate that the center was brighter than that of the surface, and the catheter looked like a graded white line. Positive inner objective visibility values indicate that the center of the catheter was darker than the edges, and the catheter appeared as white parallel lines. Subjective visibility (subjective visibility units, [SVUs]) is a measure of the recognizability of the lines on the image as a catheter. SVUs fall on a scale ranging from 0 to 3 ( 0 = invisible, 1 = poor, 2 = good, 3 = excellent), and were scored in 0.5 increments by 10 anesthesiologists. Evaluations were made based on the portion of the catheter, which was $1 \mathrm{~cm}$ deep at $30^{\circ}$ relative to the surface of the pork phantom.

\section{Statistical analysis}

Unpaired t tests were used to compare the continuous 
variables, Wilcoxon signed-rank tests were used to compare the ordinal variables, and single-factor ANOVA was used in multivariate analyses. All statistics were calculated using StatView (Version 4.54, SAS Institute Inc., Cary, NC). A P value $<0.05$ was considered statistically significant. Data are presented as the mean \pm SD.

\section{Results}

\section{Catheter}

Fig. 1 shows a sample image of each catheter. At $0^{\circ}$, the mean outer objective visibility of all catheters was significantly higher than that of the brightest tissue $(119 \pm 27$ PIU vs. $81 \pm 19$ PIU, $\mathrm{P}<0.01)$. Visibility measures for the tested catheters are shown in Fig. 2. Among the catheters tested, Perifix ONE had

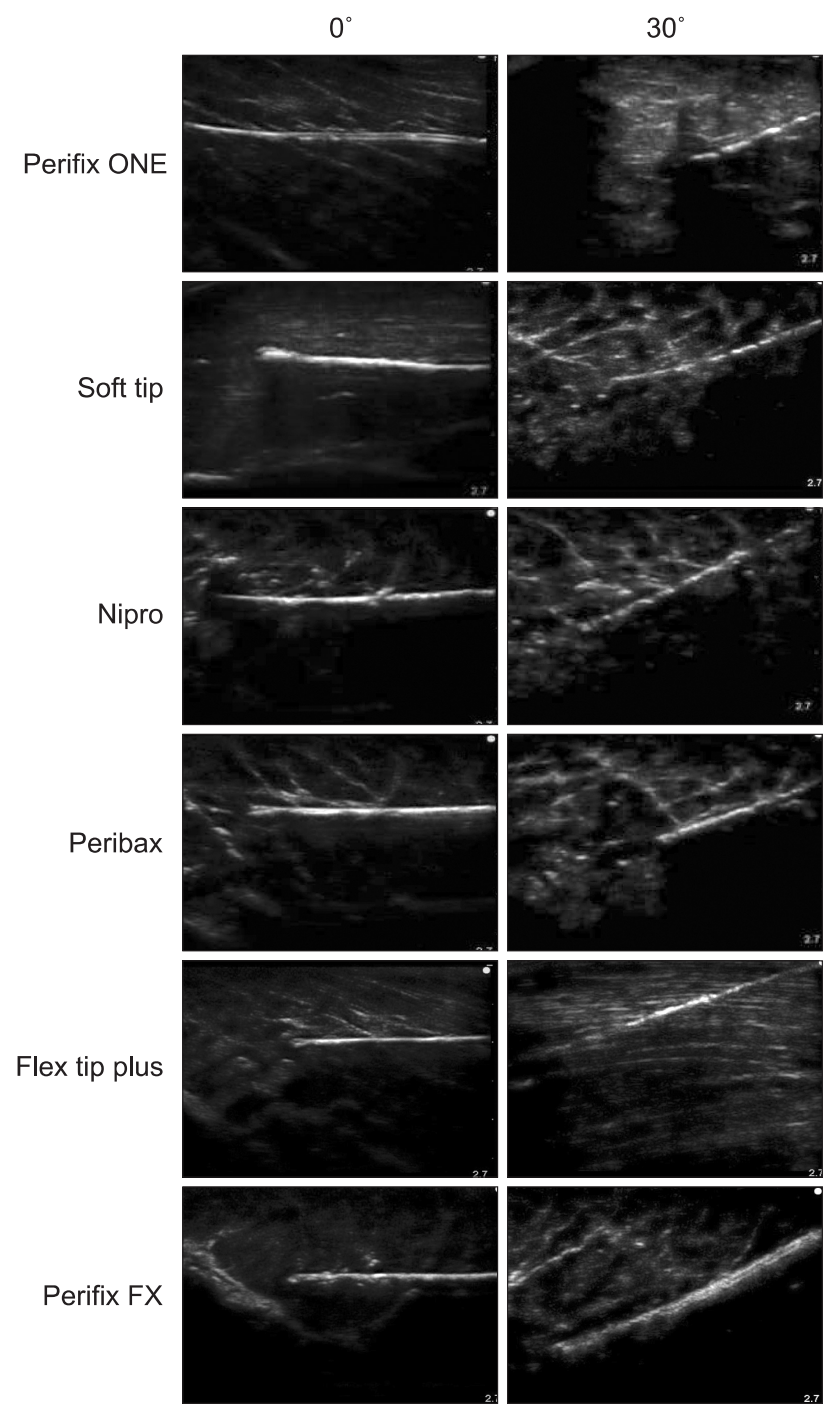

Fig. 1. A sample ultrasound image of each catheter at $0^{\circ}$ and $30^{\circ}$. the highest subjective and inner objective visibility scores. The outer objective visibility was not significantly different between the catheters. At $30^{\circ}$, the outer objective visibility of all catheters was comparable to that of the brightest tissue ( $79 \pm 16$ PIU vs. 80 \pm 17 PIU, $\mathrm{P}=0.66)$. Among the catheters tested, Perifix FX had the highest subjective and inner objective visibility. The outer objective visibility was not significantly different between the catheters (Fig. 2).

Subjective visibility was more strongly correlated with the inner objective visibility than with the outer objective visibility, at both $0^{\circ}\left(\mathrm{R}^{2}=0.79, \mathrm{P}<0.01\right.$ vs. $\left.\mathrm{R}^{2}=0.11, \mathrm{P}=0.49\right)$ and $30^{\circ}\left(\mathrm{R}^{2}=\right.$ $0.66, \mathrm{P}<0.01$ vs. $\mathrm{R}^{2}=0.21, \mathrm{P}=0.27$ ) (Fig. 3 ).

\section{Catheter diameter}

At $0^{\circ}$, there was no significant difference in any visibility measures between the catheters of different diameter. At $30^{\circ}$, subjective and inner objective visibilities of 19-gauge catheters were significantly greater than those of the 20-gauge catheters (Table 2).

\section{Angle}

All visibility measures were significantly lower at $30^{\circ}$ than at $0^{\circ}$ (subjective visibility $1.7 \pm 0.5$ SVU vs. $1.2 \pm 0.3$ SVU, $\mathrm{P}<0.01$; outer objective visibility $119 \pm 27$ PIU vs. $78 \pm 16$ PIU, $\mathrm{P}<0.01$; inner objective visibility $3.5 \pm 22$ PIU vs. $-4.9 \pm 18$ PIU, $\mathrm{P}=0.02$ ).

\section{Discussion}

\section{Catheter}

Observers were able to recognize every catheter as a catheter at both angles of the insertion. The surface brightness does not contribute to the subjective visibility, although, the needle brightness does. The positive inner objective visibility values of Perifix ONE and Perifix FX, indicate a relatively dark center, which probably helps to make it more visible at both angles. According to the manufacturer, neither of these catheters is designed for ultrasound visibility. Some particular combinations of the materials and their thickness change the reflectivity of an ultrasound beam and may enhance the dark at the center of the catheter, as reported in the study of the needle visibility, enhanced by the insertion of a guide wire [8].

\section{Catheter diameter}

Although both 19-gauge catheters are metallic, they were not any more visible at $0^{\circ}$ than the 20 -gauge non-metallic catheters. At $30^{\circ}$, the 19-gauge catheters were better visualized than the 
A

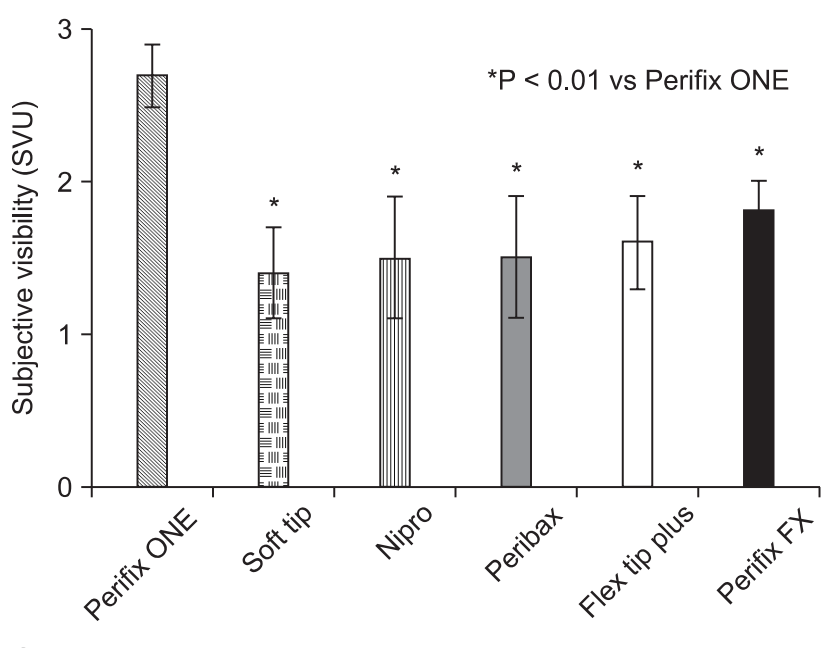

C
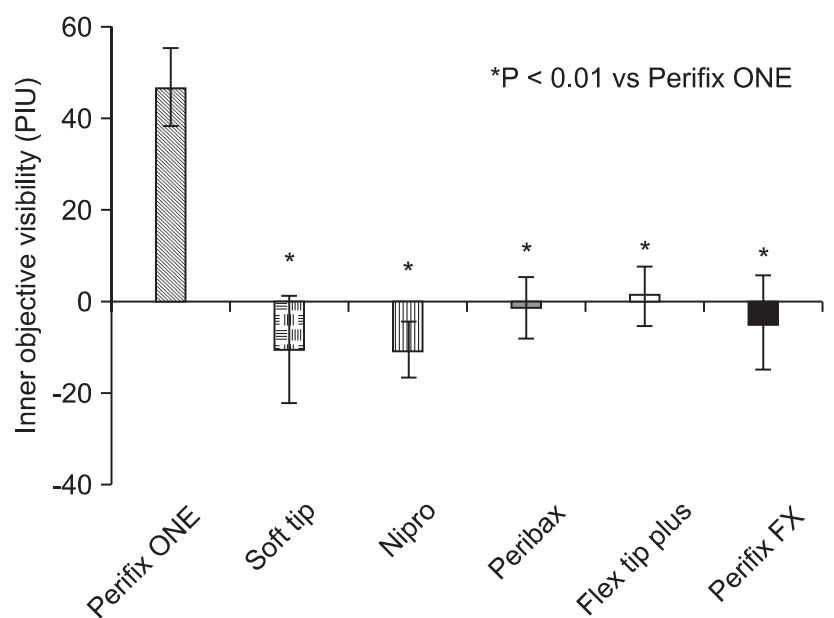

E

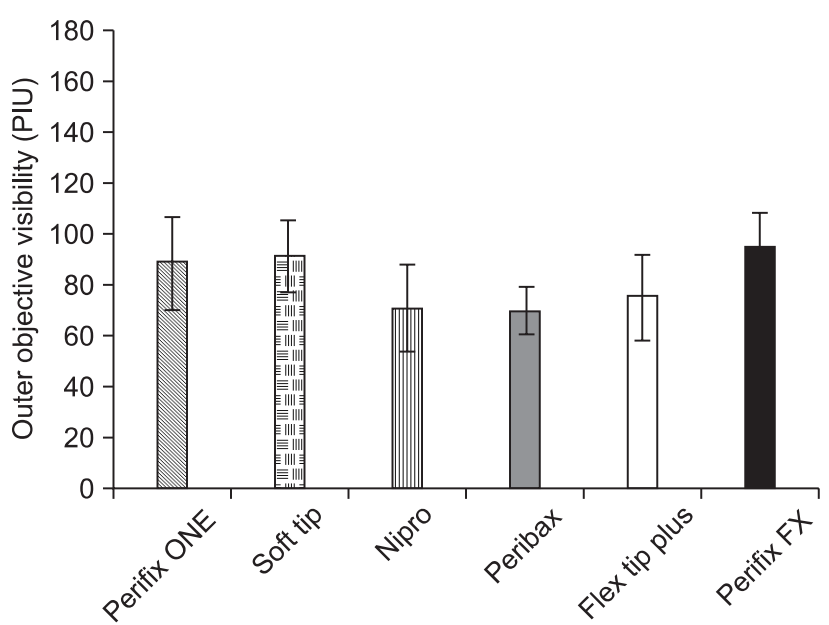

B

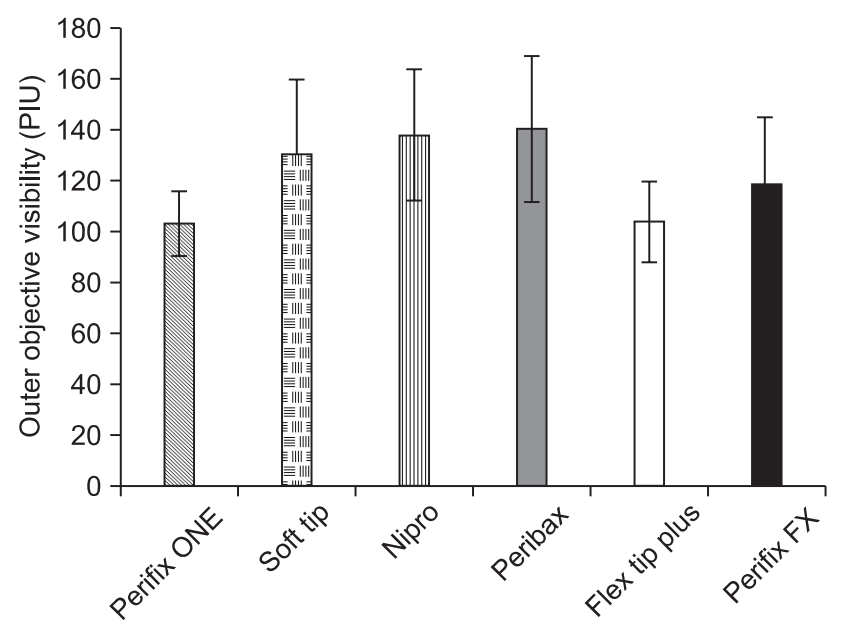

D

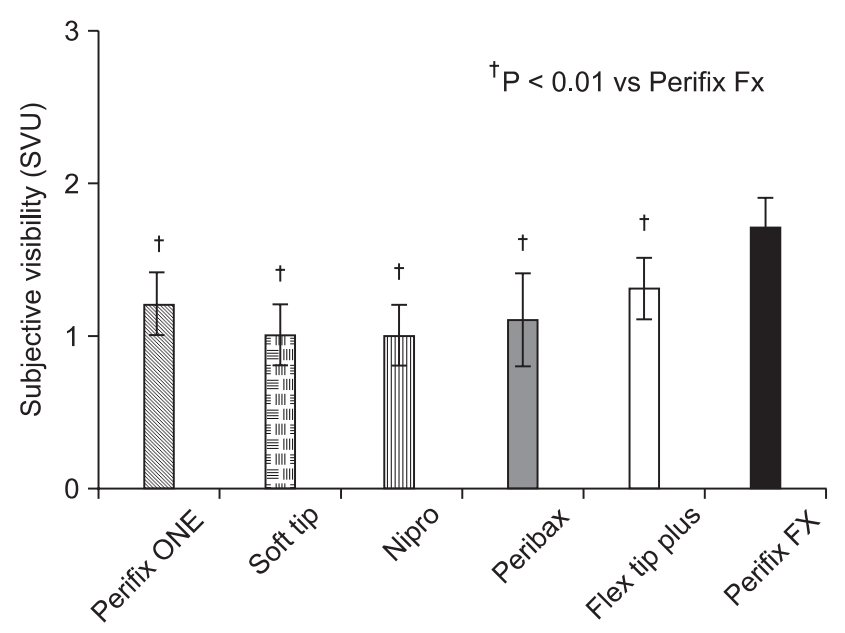

F

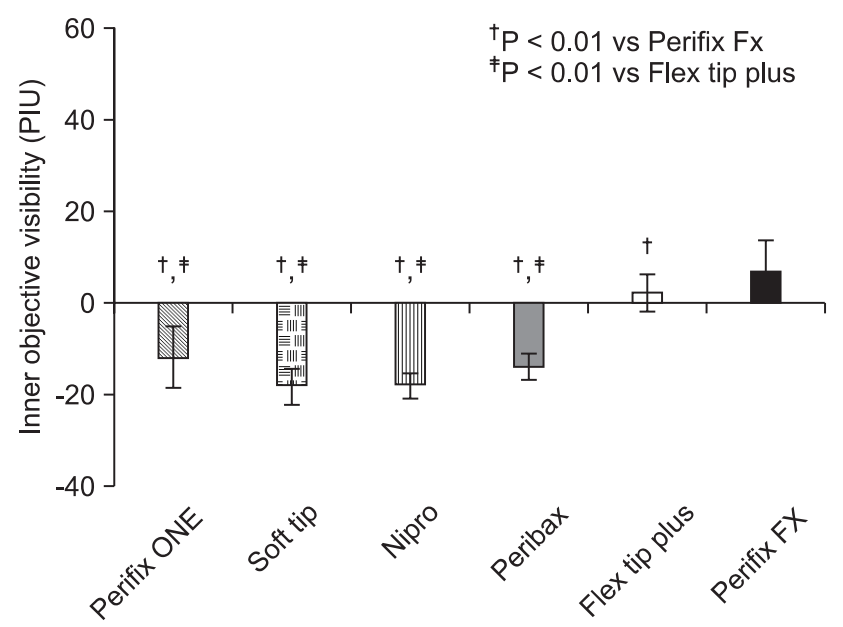

Fig. 2. Visibility of the catheters at $0^{\circ}(A-C)$ and $30^{\circ}(D-F)$. (A, D) Subjective visibility. (B, E) Outer objective visibility. (C, F) Inner objective visibility. Data are shown in mean \pm SD. $\mathrm{P}<0.05$ is considered significant. ${ }^{*}$ vs. Perifix ONE at $0^{\circ},{ }^{\dagger} \mathrm{P}$ vs. Perifix FX at $30^{\circ},{ }^{\neq} \mathrm{vs}$. Flex tip plus at $30^{\circ}$. 
A

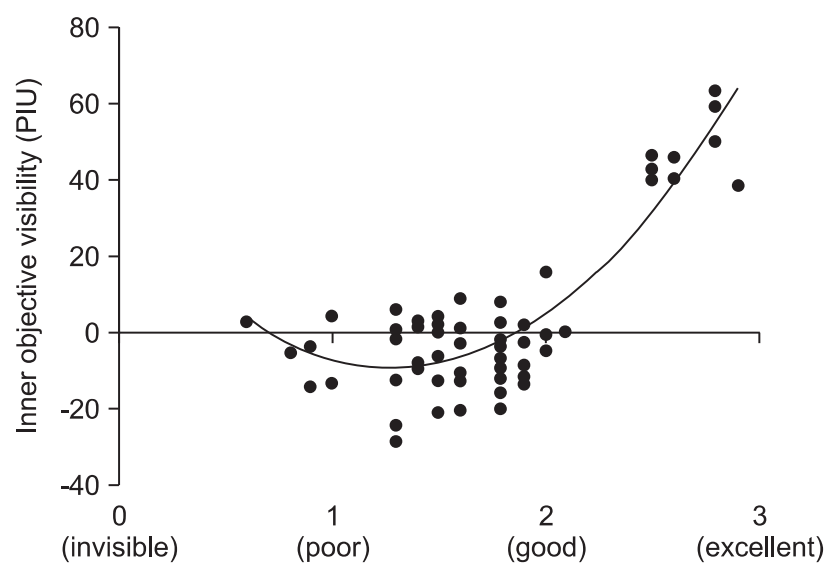

Subjective visibility (SVU)
B

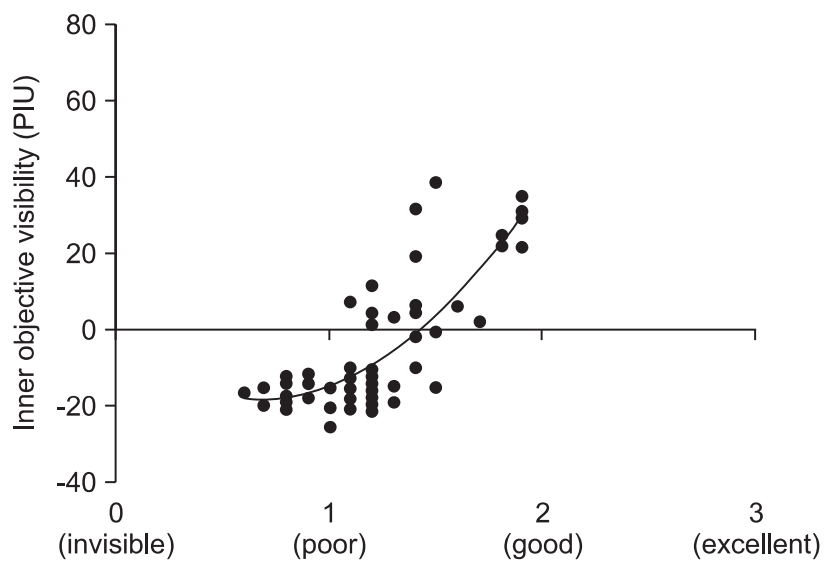

Fig. 3. The best-fit polynominal curve of subjective and inner objective visibility at $0^{\circ}(\mathrm{A})$ and at $30^{\circ}(\mathrm{B})$.

Table 2. Visibility in 19- and 20-gauge Catheters

\begin{tabular}{|c|c|c|c|c|}
\hline Angle (degrees) & Item & Diameter (gauge) & Visibility & $P$ value \\
\hline \multirow[t]{6}{*}{0} & \multirow[t]{2}{*}{ Subjective visibility (SVU) } & 19 & $1.7 \pm 0.3$ & \multirow{2}{*}{0.97} \\
\hline & & 20 & $1.7 \pm 0.4$ & \\
\hline & \multirow[t]{2}{*}{ Outer objective visibility (PIU) } & 19 & $108 \pm 21$ & \multirow{2}{*}{0.05} \\
\hline & & 20 & $114 \pm 28$ & \\
\hline & \multirow[t]{2}{*}{ Inner objective visibility (PIU) } & 19 & $-2.0 \pm 8.7$ & \multirow{2}{*}{0.17} \\
\hline & & 20 & $6.1 \pm 25$ & \\
\hline \multirow[t]{6}{*}{30} & Subjective visibility (SVU) & 19 & $1.5 \pm 0.3$ & \multirow{2}{*}{$<0.01$} \\
\hline & & 20 & $1.1 \pm 0.2^{*}$ & \\
\hline & \multirow[t]{2}{*}{ Outer objective visibility (PIU) } & 19 & $73 \pm 12$ & \multirow{2}{*}{0.10} \\
\hline & & 20 & $80 \pm 17$ & \\
\hline & \multirow[t]{2}{*}{ Inner objective visibility (PIU) } & 19 & $17 \pm 13$ & \multirow{2}{*}{$<0.01$} \\
\hline & & 20 & $-15 \pm 4.9 *$ & \\
\hline
\end{tabular}

Data are shown in mean $\pm \mathrm{SD} .{ }^{*} \mathrm{P}<0.05$ is considered significant. SVU: subjective visibility unit, PIU: pixel intensity unit.

20-gauge catheters. This difference is not due to the width of the catheter because the width did not contribute to the subjective visibility at $0^{\circ}$. The difference in the visibility between these two catheter sizes is more likely to be due to the differences in the materials used to manufacture them, as the 19-gauge catheters produced the inner objective visibility that was greater than 0 .

\section{Angle}

The brightest needle images are obtained when the ultrasound beam reflects perpendicular to the needles $[1,8]$. This is also true for the catheters, as the outer objective visibility declines as the insertion angle increases. The resulting decrease in the inner and outer objective visibility may cause an observed decline in the subjective visibility.

A non-textured needle appears as a bright straight line, with acoustic shadows on an ultrasound image [13], which is unlike any biostructure. Therefore, it is distinguishable from the surrounding tissue. However, at a steep insertion angle, visualization of non-textured needles is challenging [14-16]. Recent studies have indicated that the echogenic needles have better visibility and a higher rate of success than the nontextured needles $[17,18]$. In contrast, catheters generally appear as dark curves or short segments without acoustic shadows, and sometimes, resemble fascia, fat, or other tissues. Subjective catheter visibility is influenced by the inner objective visibility at both $0^{\circ}$ and $30^{\circ}$, and methods for enhancing the ultrasound visibility of catheters are needed.

In the present study, we did not focus on the visibility of the catheter tip, which directly affects the success of nerve blockades. However, the winding of the catheter tips make it challenging to make a distinction between them from a surface cross-section in still images. Therefore, we recommend a dynamic assessment $[19,20]$ in future studies. This study was 
limited by the placement of catheters only at the shallow sites. Placement at deeper sites results in lower surface intensities and may lead to poor inner objective visibility. We examined the visibility only at limited angles, while we found that it was difficult to delineate catheters placed at angles $>45^{\circ}$, relative to the probe surface in a preliminary study (data not shown). Although the axial or cross-section images of catheters are more easily available in clinical settings, we examined the inplane images in this study for the best possible visibility of the catheters. Our study was also limited by the inclusion of only the catheters made of different materials. No catheters with texturing or with electric stimulation are available, at present. Although the comparison between the 2 size catheters, made of the same material, would draw a more lucid conclusion, we could obtain only 6 different material catheters for this study. Comparisons among other catheter types are necessary in order to determine the best catheters for use in ultrasound-guided nerve blocks.

In conclusion, some of the catheters are recognizable when placed at either $0^{\circ}$ or $30^{\circ}$. Unlike the subjective needle visibility, the subjective catheter visibility is influenced more by the inner objective visibility than that of the outer objective visibility. For the best possible visualization, we recommend selecting a catheter with a structure that enhances the dark at the center of catheter, rather than basing catheter selection on the bore size. Placing catheters at shallow angles and as straight as possible improves visualization. This is the first report of the ultrasound visibility of catheters that are used in regional anesthesia.

\section{References}

1. Maecken T, Zenz M, Grau T. Ultrasound characteristics of needles for regional anesthesia. Reg Anesth Pain Med 2007; 32: 440-7.

2. Nichols K, Wright LB, Spencer T, Culp WC. Changes in ultrasonographic echogenicity and visibility of needles with changes in angles of insonation. J Vasc Interv Radiol 2003; 14: 1553-7.

3. Aguirre J, Blumenthal S, Borgeat A. Ultrasound guidance and success rates of axillary brachial plexus block--I. Can J Anaesth 2007; 54: 583.

4. Kapral S, Greher M, Huber G, Willschke H, Kettner S, Kdolsky R, et al. Ultrasonographic guidance improves the success rate of interscalene brachial plexus blockade. Reg Anesth Pain Med 2008; 33: 253-8.

5. Perlas A, Brull R, Chan VW, McCartney CJ, Nuica A, Abbas S. Ultrasound guidance improves the success of sciatic nerve block at the popliteal fossa. Reg Anesth Pain Med 2008; 33: 259-65.

6. Redborg KE, Antonakakis JG, Beach ML, Chinn CD, Sites BD. Ultrasound improves the success rate of a tibial nerve block at the ankle. Reg Anesth Pain Med 2009; 34: 256-60.

7. Liu SS, Ngeow JE, Yadeau JT. Ultrasound-guided regional anesthesia and analgesia: a qualitative systematic review. Reg Anesth Pain Med 2009; 34: 47-59.

8. Schafhalter-zoppoth I, Mcculloch CE, Gray AT. Ultrasound visibility of needles used for regional nerve block: an in vitro study. Reg Anesth Pain Med 2004; 29: 480-8.

9. Swenson JD, Davis JJ, DeCou JA. A novel approach for assessing catheter position after ultrasound-guided placement of continuous interscalene block. Anesth Analg 2008; 106: 1015-6.

10. Koscielniak-Nielsen ZJ, Rasmussen H, Hesselbjerg L. Long-axis ultrasound imaging of the nerves and advancement of perineural catheters under direct vision: a preliminary report of four cases. Reg Anesth Pain Med 2008; 33: 477-82.

11. Chelly JE, Casati A. Perineural infusion of local anesthetics: "more to the review". Anesthesiology 2007; 106: 191.

12. Hocking G, Hebard S, Mitchell CH. A review of the benefits and pitfalls of phantoms in ultrasound-guided regional anesthesia. Reg Anesth Pain Med 2011; 36: 162-70.

13. Chapman GA, Johnson D, Bodenham AR. Visualisation of needle position using ultrasonography. Anaesthesia 2006; 61: 148-58.

14. Culp WC, McCowan TC, Goertzen TC, Habbe TG, Hummel MM, LeVeen RF, et al. Relative ultrasonographic echogenicity of standard, dimpled, and polymeric-coated needles. J Vasc Interv Radiol 2000; 11: 351-8.

15. Bradley MJ. An in-vitro study to understand successful free-hand ultrasound guided intervention. Clin Radiol 2001; 56: 495-8.

16. Edgcombe H, Hocking G. Sonographic identification of needle tip by specialists and novices: a blinded comparison of 5 regional block needles in fresh human cadavers. Reg Anesth Pain Med 2010; 35 : 207-11.

17. Deam RK, Kluger R, Barrington MJ, McCutcheon CA. Investigation of a new echogenic needle for use with ultrasound peripheral nerve blocks. Anaesth Intensive Care 2007; 35: 582-6.

18. Hopkins RE, Bradley M. In-vitro visualization of biopsy needles with ultrasound: a comparative study of standard and echogenic needles using an ultrasound phantom. Clin Radiol 2001; 56: 499502 .

19. Hebard S, Hocking G. Echogenic technology can improve needle visibility during ultrasound-guided regional anesthesia. Reg Anesth Pain Med 2011; 36: 185-9.

20. Ponde VC, Desai AP, Shah DM, Johari AN. Feasibility and efficacy of placement of continuous sciatic perineural catheters solely under ultrasound guidance in children: a descriptive study. Paediatr Anaesth 2011; 21: 406-10. 